$8^{\text {th }}$ International conference on Research in Engineering, Science and Technology

Paris, France

November 2-4, 2018

\title{
The Magic of Shrooms: From Ancient Cultures to Modern Neurology
}

\section{Gamer AlHassan}

This research explores the relationship between psilocybe cubensis also commonly known as psilocybin, the active ingredient in "magic mushrooms"-and the formation and alteration of neural networks as well as the possible influence upon neurogenesis. Psilocybin has been classified by the U.S government as an illegal substance since the 1960s, effectively curbing most research on the drug. Attitudes towards psilocybin in the rest of the world have varied, many cultures have used the drug for centuries in religious and medical endeavors. Recently, research has shown that psilocybin has profound influences on the brain outside of its hallucinogenic effects, rather, neurotransmitters are heavily excited by the introduction of this chemical into the body. Due to this phenomenon the question of whether or not psilocybin can be used for generating new neural networks and hippocampal neurons arose. This gave a new perspective on the once illegal substance that can be potentially influential in neuromedical research. The organization of this research takes into account a multidisciplinary angle of both the physiological and anthropological influences of psilocybin. Physiologically, this research seeks to understand and further the connections between psilocybin and its effect on the human brain, specifically the alteration of neural networks and its probability of being a catalytic agent of neurogensis. While also taking into account the anthropological perspective dealing with societal behaviors towards psilocybin and the dichotomy of stigmatization and appreciation throughout different cultures. Implications of this project highlight the potential medical use of psilocybin while also respecting its cultural significance. 
